\title{
Efficacy and safety of artemether-lumefantrine and dihydroartemisinin-piperaquine in the treatment of uncomplicated Plasmodium falciparum malaria in Kenyan children aged less than five years: results of an open-label, randomized, single-centre study
}

Bernhards R Ogutu ${ }^{1,2^{*}}$, Kevin O Onyango ${ }^{1}$, Nelly Koskei ${ }^{1}$, Edgar K Omondi ${ }^{1}$, John M Ongecha ${ }^{3}$, Godfrey A Otieno ${ }^{1,2}$, Charles Obonyo ${ }^{3}$, Lucas Otieno ${ }^{1,2}$, Fredrick Eyase ${ }^{2}$, Jacob D Johnson ${ }^{2}$, Raymond Omollo ${ }^{4}$, Douglas J Perkins ${ }^{5}$, Willis Akhwale $e^{6}$ and Elizabeth Juma ${ }^{1,7}$

\begin{abstract}
Background: This open-label, randomized study evaluated efficacy and safety of artemether-lumefantrine (AL) and dihydroartemisinin-piperaquine (DP) in treatment of uncomplicated falciparum malaria in children below five years of age, to build evidence on use of AL as first-line treatment and DP as second-line treatment in Kenya.

Methods: A total of 454 children aged six to 59 months with uncomplicated falciparum malaria were randomized (1:1) to receive AL dispersible or DP paediatric tablets and followed up for 42 days. Primary efficacy variable was corrected adequate clinical and parasitological response (ACPR) rate on day 28. Secondary variables included corrected (day 14 , 28 and 42), uncorrected (day 3, 14, 28 and 42) cure rates, parasitological failure at days 3, 14 and 42. Acceptability and tolerability of both drugs were assessed by caregiver questionnaire.

Results: On day 28, corrected ACPR rates for AL dispersible and DP paediatric were 97.8\% (95\% Cl: 94.9-99.3) and 99.1\% (95\% Cl: 96.8-99.9), respectively, in intention-to-treat population, with no significant treatment differences noted between AL dispersible and DP paediatric arms. Additionally, no significant differences were observed for PCR corrected cure rates on days 14 and ACPR on day 42 for AL dispersible (100\%; 96.8\%) and DP paediatric (100\%; 98.7\%). Similarly, for PCR uncorrected cure rates, no significant differences were seen on days 3, 14, 28, and 42 for AL dispersible (99.1\%; 98.7\%; 81.1\%; $67.8 \%)$ and DP paediatric (100\%; 100\%; 87.7\%; 70.5\%). Parasite clearance was rapid, with approximately $90 \%$ clearance achieved in 40 hours in both treatment arms. Incidence of adverse events was related to underlying disease; malaria being reported in both treatment arms. One serious adverse event was noted in AL dispersible (0.42\%) arm, not related to study drug. Adherence to treatment regimen was higher for children treated with AL dispersible (93.6\%) compared to DP paediatric (85.6\%). Acceptability of AL dispersible regimen was assessed as being significantly better than DP paediatric. (Continued on next page)
\end{abstract}

\footnotetext{
* Correspondence: bernhards.ogutu@usamru-k.org

${ }^{1}$ Centre for Clinical Research, Kenya Medical Research Institute, Kisumu,

Kenya

${ }^{2}$ Walter Reed Project/Centre for Clinical Research, Kenya Medical Research

Institute, Kisumu, Kenya

Full list of author information is available at the end of the article
} 
(Continued from previous page)

Conclusions: AL and DP were both efficacious and well tolerated, and had similar effects at day 42 on risk of recurrent malaria. No signs of Plasmodium falciparum tolerance to artemisinins were noted.

Trial registration: PACTR201111000316370.

Keywords: Artemether-lumefantrine, Dihydroartemisinin-piperaquine, Uncomplicated Plasmodium falciparum malaria

\section{Background}

Malaria continues to be a major health problem worldwide, with an estimated 216 million cases in 2010, approximately 174 million of which were in the African region [1]. Children less than five years of age are at increased risk of Plasmodium falciparum malaria, with a reported $86 \%$ mortality rate globally [1]. Artemisinin-based combination therapy (ACT) is recommended as the first-line treatment for uncomplicated P. falciparum malaria by the World Health Organization (WHO) [2], over chloroquine and sulpha drugs worldwide. Multidrug resistance has been reported for monotherapy (for example artemisinin [3-5]) and some of the available combination chemotherapy (for example, sulphadoxine/sulphalene-pyrimethamine [6]) used for malaria. Treatment with ACT is known to improve cure rates, results in rapid parasite clearance [2,7] and reduces gametocyte carriage resulting in a decrease in parasite transmission $[2,8-10]$.

Artemether-lumefantrine (AL, Coartem ${ }^{\odot}$, Novartis Pharma AG) is a first fixed-dose ACT, which meets the WHO prequalification criteria for efficacy, safety and quality, and is indicated for the treatment of uncomplicated falciparum malaria, or mixed infections including $P$. falciparum in adults, children, and infants $(>5 \mathrm{~kg}$ body weight). Since the prequalification of Coartem, other AL generics have been prequalified by WHO. In clinical studies, AL demonstrated consistent 28-day polymerase chain reaction (PCR)-corrected cure rates of more than $95 \%$ in adult and paediatric populations with a favourable safety and tolerability profile [11]. With rapid parasite clearance and gametocyte reduction also being reported [11], the widespread adoption of AL has led to a significant contribution in the reduction of malaria burden in Sub-Saharan Africa [12]. Since the Kenyan Ministry of Health introduced AL as the firstline treatment for uncomplicated falciparum malaria in 2006, there has been a notable reduction in child mortality rates [13]. Novartis launched the paediatric formulation of AL (AL dispersible) in 2009, developed jointly with Medicines for Malaria Venture (MMV). It is a sweet-tasting and easy-to-administer tablet which is now approved in over 40 countries. AL dispersible has proven in clinical trials to be as well tolerated and as effective as regular AL tablets $[14,15]$. Moreover, it has been shown that standard African diets (generally consisting of a carbohydrate staple supplemented by pulses, nuts, meat or fish and fat from oil crops or from vegetables or plants) and milk are adequate to ensure AL efficacy [16]. However, AL is associated with a high risk of reinfection soon after therapy in high-transmission areas [17].

Dihydroartemisinin-piperaquine (DP: Duo-cotecxin ${ }^{\oplus}$, Holley-Cotec Pharmaceuticals) is an ACT that is administered as a single daily dose over three days and has been shown to be well tolerated and highly effective against falciparum malaria [18]. In a previous comparative assessment, DP has been proven to be as efficacious and safe as AL in the treatment of uncomplicated falciparum malaria in Zambian children less than five years of age. DP has been suggested for use as a rescue and/or alternative treatment to $\mathrm{AL}$, or as second-line treatment following treatment failure with the initial treatment [19]. The Kenyan Ministry of Health adopted DP as second-line treatment in 2009.

To further build on the evidence from earlier studies that used AL as a first-line and DP as second-line treatment, this study evaluated the efficacy and safety of AL dispersible $v s$ DP paediatric in Kenyan children less than five years of age for the treatment of uncomplicated falciparum malaria.

\section{Methods}

This was an open-label, randomized, single-centre study (Trial registration number: PACTR201111000316370). The protocol was approved by the Kenya Medical Research Institute Ethics Review Committee. The study was conducted in accordance with the Declaration of Helsinki (2002), Good Clinical Practices guidelines set up by the International Conference on Harmonization [20], and local applicable laws and regulations.

\section{Patients}

Children aged six to 59 months (inclusive), weighing $5 \mathrm{~kg}$ or more with fever $\geq 37.5^{\circ} \mathrm{C}$ (axillary), who presented to the Ombeyi Dispensary with probable clinical malaria, mono-infection with $P$. falciparum at an asexual parasite density of 1,000-200,000 parasites $/ \mu \mathrm{L}$, and who were able to take drug orally, were eligible for enrolment in the study. Patients were excluded if they had severe and/or complicated malaria [21], including severe anaemia (haemoglobin $(\mathrm{Hb}) \leq 5 \mathrm{~g} / \mathrm{dL}$ ), experienced two or more seizures in the previous 24 hours and hyperparasitaemia 
(parasites $>200,000 / \mu \mathrm{L}$ ), or had a general clinical condition requiring hospitalization. Patients who had concomitant infections/disease at the time of presentation, with past or present history of chronic illnesses or any other underlying illness that would compromise the diagnosis and evaluation of the response to the study drug, with a history of allergy to artemisinin, lumefantrine or piperaquine were excluded. Patients undergoing full treatment with other anti-malarial drugs within the previous 14 days were also excluded. Eligible patients were enrolled after the parent/guardian signed the written informed consent.

\section{Study design}

The study comprised a three-day treatment period (day 1 taken as the first day of treatment) and 42-day follow-up. Eligible patients were randomized to one of the two treatment arms (1:1) to receive either AL dispersible (six doses/three days) or DP paediatric (three doses/three days). Patients in the AL dispersible arm with body weight $5-14 \mathrm{~kg}$ received one tablet (artemether $20 \mathrm{mg}$, lumefantrine $120 \mathrm{mg}$ ) per dose, and 15-24 kg received two tablets per dose. Patients in the DP paediatric arm received the standard dosage of $2.25 \mathrm{mg} / \mathrm{kg}$ and $18 \mathrm{mg} / \mathrm{kg}$ per dose of dihydroartemisinin and piperaquine, respectively, rounded up to the nearest half tablet. The drugs were dispersed in a small volume of water or milk and administered by the parents/caregivers under the observation of the study personnel. If there was vomiting within 30 minutes of the dose then the child was re-dosed and if the child vomited the replacement dose, he/she was dropped from the study. All the drug administration occurred in an inpatient setting. Children who developed severe malaria were treated with quinine as per the national guidelines.

Children were admitted to hospital for the first three days for observed treatment and close monitoring. They were evaluated daily in the ward and eight-hourly blood slides were performed until two consecutive negative blood smears for asexual falciparum malaria were obtained. Children were discharged after they had a negative slide and were clinically stable. At discharge, the parent/caregiver completed a questionnaire on the treatment acceptability, and the children were followed up on days 7, 14, 28, 42 and any other day the child was unwell. During the visits, a blood slide was analysed for parasite quantification; blood was collected for a complete blood count $(\mathrm{CBC})$ and a filter paper sample for genotyping in case of parasite reappearance; physical examination was performed and vital signs, axillary temperature were recorded. Adverse events (AEs) and serious adverse events (SAEs) were recorded and monitored throughout the study.

\section{Assessments}

The primary efficacy assessment included patients with PCR-corrected parasitaemia by day 28 , i e, patients with an adequate clinical and parasitological response (ACPR). According to the WHO definition [22], ACPR is the absence of parasitaemia on day 28 or day 42 irrespective of axillary temperature, without previously meeting any of the early treatment failure (ETF), late treatment failure (LTF) or late parasitological failure (LPF) criteria. As per the WHO definition [22], ETF is development of danger signs of malaria or severe malaria on post treatment days $0,1,2$ or 3 , with evidence that the patient is symptomatic and parasitaemic to a greater degree than the value recorded at entry to the trial on day 2; parasitaemic on day 3 greater than $25 \%$ of the value recorded on entry to the trial and with axillary temperature $\geq 37.5^{\circ} \mathrm{C}$. LTF is development of danger signs or severe malaria between post-treatment days 4 to 14 inclusive when: a) the patient has not previously met the criteria for ETF and, b) PCR analysis of markers including merozoite surface protein (MSP)-1, MSP-2 and glutamaterich protein (GLURP) suggests that the parasites are unlikely to be a new infection, with evidence that the patient is symptomatic and any level of $P$. falciparum parasitaemia on any day between 4, 28 or day 42, inclusive with axillary temperature $\geq 37.5^{\circ} \mathrm{C}$ in patients not meeting ETF criteria earlier. LPF is presence of parasitaemia on any day between day 7 and day 28 (or day 42) with axillary temperature $<37.5^{\circ} \mathrm{C}$ in patients who did not previously meet any of the criteria of ETF or late clinical failure.

The secondary efficacy and safety assessments included parasitological failure at days 3, 14 and 42, (defined as presence or absence of parasitaemia on assessment), determination of the level of adherence, ease of use, acceptability and adverse events (serious and non-serious) reported as an indicator of safety for both drugs among patients enrolled in the study.

Safety assessments included recording of adverse events (AEs, by system organ class and preferred terms), serious adverse events (SAEs), treatment emergent serious and non-serious adverse events (AEs occurring between day 1 and 35 of treatment period or AEs with unknown onset dates), and collection of clinical laboratory data for haematology and blood chemistry. According to the ICH guidelines [20], AEs are defined as any untoward medical occurrences in a patient administered a pharmaceutical product and which does not necessarily have a causal relationship to the treatment. SAEs are untoward medical occurances that at any dose, result in death, are life threatening, require hospitalization, prolongation of existing hospitalization or result in persistant, and significant disability, or is a congenital anomaly/birth defect. During the study period the area was under indoor residual spraying (IRS) for malaria as a pilot district in the deployment of IRS in high transmission areas by the Division of 
Malaria Control of the Ministry of Public Health and Sanitation, Kenya.

\section{Statistical analysis}

In this non-inferiority trial, it was estimated that 193 patients per arm were needed to complete the study, assuming that at day 28 , the efficacies of AL dispersible and DP paediatric were $90 \%$, with a power of $80 \%$, and a two-sided significance level of 5\%. Allowing for a 10\% dropout rate, it was calculated that 233 patients per arm (426 in total) had to be randomized. Baseline data were summarized by continuous variables using mean (standard deviation (SD)) if normally distributed, tested using t-test or ANOVA where appropriate or using median and inter-quartile range and non-parametric testing if not normally distributed.

The efficacy variables were analysed on the intent-totreat (ITT) and per protocol (PP) population. The ITT population included all patients who received at least one dose of study drug. Patients with a major protocol deviation were included in the analyses. The PP population excluded patients with predefined protocol violations and included patients who completed all visits as specified in the protocol; had no major protocol violation with regards to inclusion/exclusion criteria; did not take any prohibited concomitant medications during the treatment period. Patients who were withdrawn from the study due to an $\mathrm{AE}$ or lack of efficacy, or who were considered a treatment failure, were included in the PP population.

For the primary efficacy variable, the treatment effect was the difference in efficacy between the two arms at day 28. Missing data was analysed by using complete case analysis wherein patients with missing efficacy data were excluded from the analysis. For secondary efficacy analyses, the treatment effect was the difference in efficacy between the two treatment arms at days 3, 14 and 42. In the analyses of safety data, the biological parameter analysis by treatment regimen was assessed by the mean (95\% confidence interval (CI)) change from baseline on day 28. The mean (SD) parameter value at day 28 was presented for each arm. Formal testing for a difference between treatments was done using ANOVA comparing average day 28 values, adjusting for baseline values. Adverse events (both serious and non-serious) have been tabulated using preferred terms by treatment arm in Medical MeDRA V. 12. Failure rate has also been presented based on parasite density at baseline.

\section{Results}

This study was conducted at Ombeyi dispensary in Nyando District, Kenya. The first patient was enrolled on 5 March, 2010 and the last patient completed the study on 30 November, 2011.

\section{Patient disposition, demographics, and baseline characteristics}

A total of 1979 children were screened of which 1525 were screening failures, with most being non-malaria cases, low parasite density, mixed species malaria infections and consent refusal (Figure 1). In total, 454 patients were randomized (1:1) to the AL dispersible or DP paediatric treatment arms and 448 (98.6\%) completed the study. Two patients receiving DP and four patients receiving $\mathrm{AL}$ dispersible treatment were withdrawn from

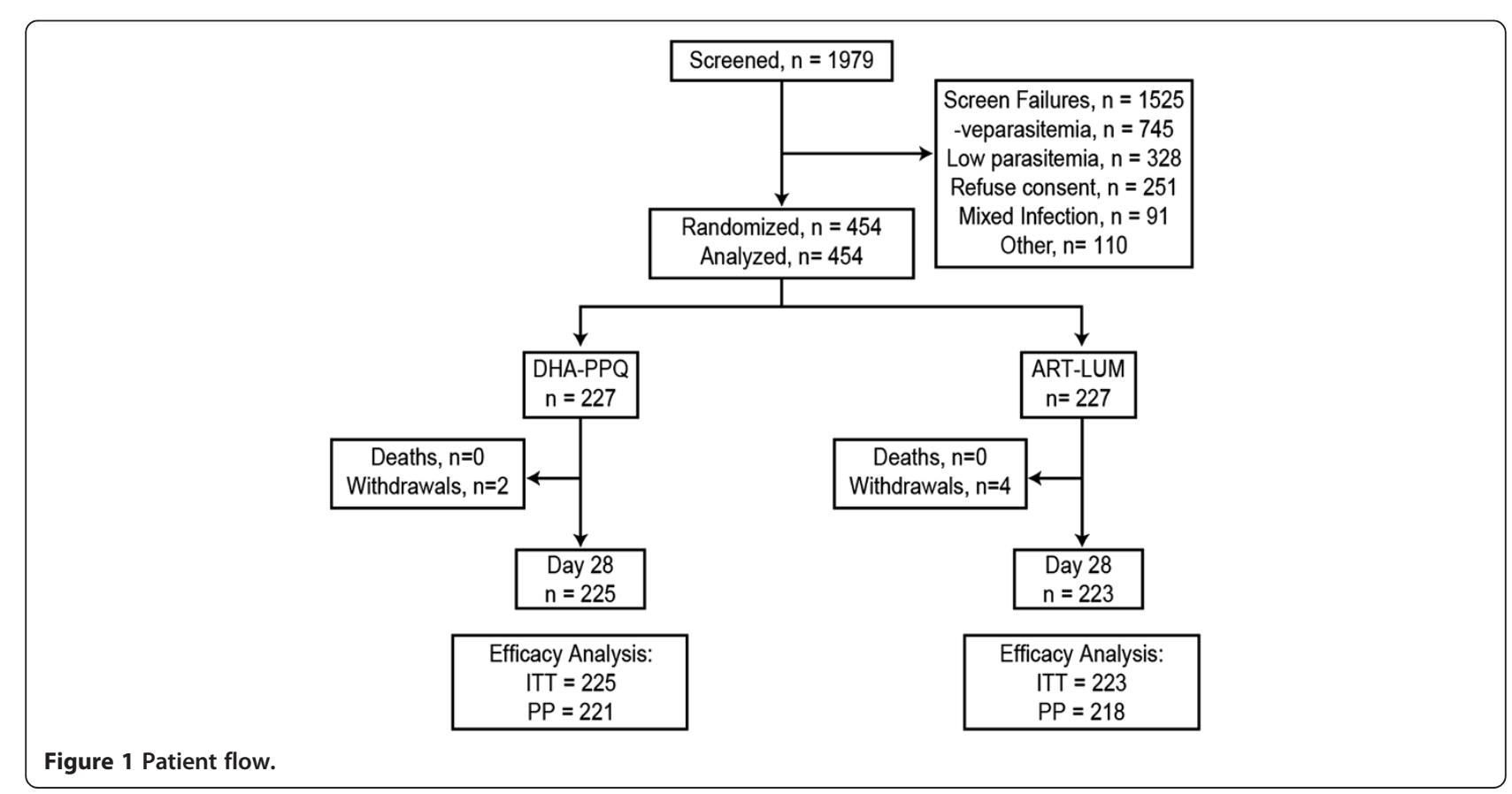


Table 1 Patient demographics and baseline characteristics

\begin{tabular}{lccc}
\hline Characteristics & AL dispersible $(\mathbf{n}=\mathbf{2 2 7})$ & DP paediatric $(\mathbf{n}=\mathbf{2 2 7})$ & $\mathbf{p}$ value \\
\hline Age $($ months) & $29.6(15.2)$ & $32.0(14.9)$ & 0.094 \\
Weight $(\mathrm{kg})$ & $12.2(3.2)$ & $12.3(3.2)$ & 0.709 \\
Temperature $\left({ }^{\circ} \mathrm{C}\right)$ & $38.3(1.0)$ & $38.3(2.1)$ & 0.949 \\
Parasite density $(/ \mu \mathrm{L})$, mean $(95 \% \mathrm{Cl})^{\dagger}$ & $38,202.2(32,180.0$ to $45,351.3)$ & $38,545.7(32,615.8$ to $45,553.8)$ & $0.941^{\ddagger}$ \\
Haemoglobin $(\mathrm{g} / \mathrm{dL})$ & $9.2(1.9)$ & $9.4(2.0)$ & 0.293 \\
White blood cells $\left(\times 10^{3} / \mu \mathrm{L}\right)$ & $9.9(4.5)$ & $9.5(3.8)$ & 0.258 \\
Platelets $\left(\times 10^{3} / \mu \mathrm{L}\right)$ & $134.8(66.1)$ & $34.9(67.1)$ & 0.988 \\
Red blood cells $\left(\times 10^{6} / \mu \mathrm{L}\right)$ & $3.7(0.9)$ & $43.1(17.8)$ & 0.764 \\
Neutrophils $(\%)$ & $41.0(17.5)$ & $48.4(17.5)$ & 0.203 \\
Lymphocytes $(\%)$ & $50.6(17.2)$ & $7.6(4.9)$ & 0.182 \\
Monocytes $(\%)$ & $7.4(3.0)$ & 0.559 \\
\hline D.
\end{tabular}

Data are mean $( \pm S D)$ unless otherwise specified. AL: Artemether-lumefantrine; DP: dihydroartemisinin-piperaquine; ${ }^{*} p$ value from t-test or Mann-Whitney test;

${ }^{\dagger}$ Geometric mean with its $95 \% \mathrm{Cl}^{\ddagger}{ }^{\ddagger} \mathrm{p}$ value from t-test on the log parasite density.

the study by the investigator after day 1 due to inability to tolerate the medication (vomiting; Figure 1). The baseline demographics and clinical characteristics were similar between the two arms (Table 1).

The corrected ACPR rates in the ITT population were 97.8\% (95\% CI 94.9-99.3) and 99.1\% (95\% CI 96.8-99.9) for AL dispersible and DP paediatric, respectively, on day 28 (treatment difference 1.3\%, 95\% CI: $-1.0-3.6 \%$; $\mathrm{p}=0.258$; Figure 2a). No significant treatment differences were observed for the corrected ACPR rates on days 14 and 42 between the AL dispersible and the DP paediatric arm in the IIT population (Figure 2a). For the uncorrected cure rates, no significant differences were seen on days 3,14 and 28 and 42 between the AL dispersible and the DP paediatric arm $(p>0.05)$ for all comparisons (Figure 2b). Similar results were obtained
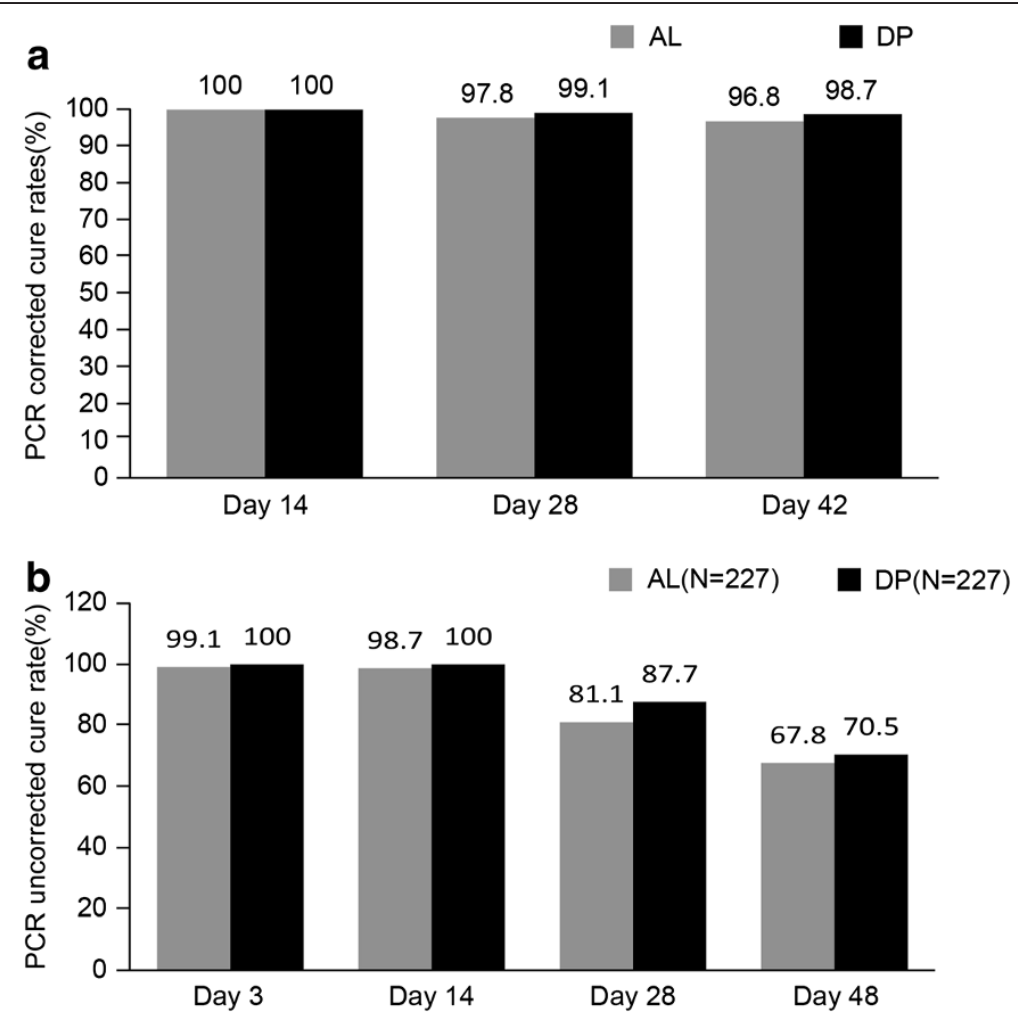

Figure 2 PCR-corrected and uncorrected cure rates in ITT population. a) PCR-corrected cure rates in ITT population. b) PCR-uncorrected cure rates in ITT population. AL: Artemeter-lumefantrine dispersible; DP: dihydroartemisinin-piperaquine paediatric, ITT: intension to treat. 


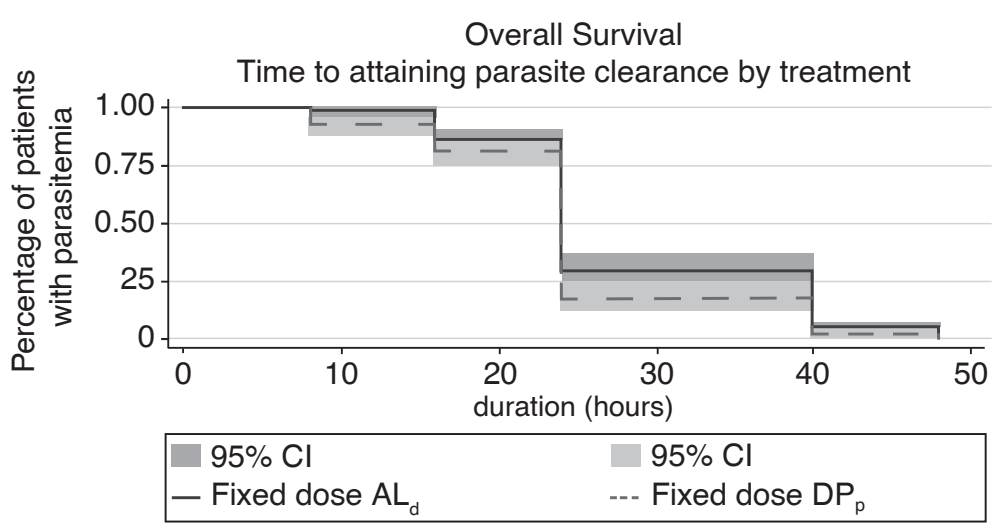

Figure 3 Parasite clearance time following ACT treatment for malaria.

in the PP population for corrected and uncorrected ACPR rates (Figure 2).

Parasite clearance was rapid, with $90 \%$ clearance achieved in 40 hours in both treatment arms (Figure 3), and $50 \%$ parasite clearance was achieved within 24 hours in both treatment arms. Treatment failure at day 28 was unaffected by baseline parasite load for both AL dispersible and DP paediatric arms. The treatment failure rate for AL dispersible and DP paediatric was comparable for parasite densities of $<50,000 / \mu \mathrm{L}$ and $>50,000 / \mu \mathrm{L}$ (23 vs 20 for AL dispersible, $\mathrm{p}=0.634 ; 12$ s 14 for DP paediatric; $\mathrm{p}=0.959$; Table 2). Similar results were observed for parasite rates $<100,000$ and $>100,000$ (31 vs 12 for AL; $\mathrm{p}=0.901 ; 23$ vs 5 for DP; $\mathrm{p}=0.379$ ).

\section{Safety}

The overall incidence of AEs was 65.5\% (156/238) and $67.5 \%(156 / 231)$ in the AL dispersible and DP arms, respectively (Table 3 ). The most frequently reported AEs were related to the underlying disease; malaria was reported in both treatment arms $(25.6 \%$ and $18.2 \%$ in the AL dispersible and DP paediatric arms, respectively). Cough was predominant in both treatment arms $(15.5 \%$ and $17.3 \%$ in the AL dispersible and DP paediatric arms, respectively). One patient in the AL dispersible arm had a SAE, severe malaria, not considered by the investigator to be related to the study drug. No deaths were reported during the study. Both treatments were generally well tolerated. The safety profile was comparable for AL dispersible and DP paediatric arms with regard to biological parameters including haemoglobin, white blood cells, platelets, red blood cells (RBCs), lymphocytes and monocytes (Table 4).

Tolarability and acceptability assessed by caregiver questionnaire, including general questions with respect to preferred paediatric formulations, are presented in Table 5. Adherence to treatment regimen was higher in the AL dispersible arm (93.6\%) compared to DP paediatric (85.6\%). Among the 126 patients randomized to receive AL dispersible, 103 (82\%) considered it 'simple' or 'very simple' to use compared with $83(67 \%)$ in the DP paediatric arm. The taste of AL dispersible was 'liked' or 'liked very much' by $72 \%$ of respondents, compared with $56 \%$ for DP paediatric. The majority in both groups took the drug with a meal (AL dispersible $=94.4 \%$; DP paediatric $=89.4 \%$ ), and preferred water to dissolve the tablets $(\mathrm{AL}$ dispersible $=94.4 \%$; DP paediatric $=89.4 \%$ ). In general, caregivers preferred the dispersible tablet formulation (drug given as tablet dissolved in a small volume of water/milk) as compared to a syrup formulation (AL dispersible $=76.8 \%$ vs $16.8 \%$; DP paediatric $=62.3 \%$ vs $29.5 \%)$.

\section{Discussion}

In the present study, AL dispersible and DP paediatric were found to be highly efficacious for the treatment of uncomplicated falciparum malaria in Kenyan children less than five years of age. The 28-day corrected ACPR

Table 2 Effect of parasite load at baseline on failure: day 28

\begin{tabular}{|c|c|c|c|c|c|c|}
\hline Parasite load at baseline & Failure at day 28 & p-value* & AL dispersible & p-value* & DP dispersible & p-value* \\
\hline$\leq 50,000(n=223)$ & $37(16.6 \%)$ & 0.796 & $23(20.5 \%)$ & 0.735 & $14(12.6 \%)$ & 0.959 \\
\hline$>50,000(n=219)$ & $34(15.5 \%)$ & & $20(18.2 \%)$ & & $14(12.6 \%)$ & \\
\hline$\leq 100,000(n=328)$ & $54(16.5 \%)$ & 0.768 & $31(19.1 \%)$ & 0.851 & $23(13.9 \%)$ & 0.484 \\
\hline$>100,000(n=114)$ & $17(14.9 \%)$ & & $12(20.0 \%)$ & & $5(9.3 \%)$ & \\
\hline
\end{tabular}


Table 3 Most frequent adverse events ( $\geq 2 \%$ in any treatment arm)

\begin{tabular}{|c|c|c|}
\hline Adverse events & $\begin{array}{l}\text { AL dispersible } \\
(\mathrm{n}=238)\end{array}$ & $\begin{array}{l}\text { DP paediatric } \\
(\mathrm{n}=231)\end{array}$ \\
\hline $\begin{array}{l}\text { Patients with at least one adverse } \\
\text { event }\end{array}$ & $156(65.54)$ & $156(67.53)$ \\
\hline Malaria & $61(25.63)$ & $42(18.18)$ \\
\hline Cough & $37(15.55)$ & $40(17.32)$ \\
\hline Anaemia & $10 \quad(4.20)$ & $8 \quad(3.46)$ \\
\hline Fever & $7 \quad(2.94)$ & $14 \quad(6.06)$ \\
\hline Tinea capitis & $10 \quad(4.20)$ & $12 \quad(5.19)$ \\
\hline Rhinitis & $4 \quad(1.68)$ & $13 \quad(5.63)$ \\
\hline Gastroenteritis & $9 \quad(3.78)$ & (2.16) \\
\hline Loss of appetite & $3(1.26)$ & (2.59) \\
\hline Otitis media & $7 \quad(2.94)$ & (2.16) \\
\hline
\end{tabular}

AL: Artemether-lumefantrine; DP: dihydroartemisinin-piperaquine.

rates in patients receiving AL dispersible were not significantly different to those seen in patients receiving DP paediatric. The corrected ACPR rates were similar to those previously reported in Kenya and other parts of Africa [19,22]. This shows that despite scaled-up use of AL dispersible in Kenya, the AL is still efficacious as first-line treatment. In contrast to earlier findings $[19,23,24]$, there were no significant treatment differences noted in uncorrected ACPR rates between the AL dispersible and DP paediatric arms at any of the time points assessed, despite the difference in half-life between lumefantrine (four to six days) $[25,26]$ and piperaquine (two to three weeks) [27]. This may be due to the intensity of transmission in this region.

The current study was conducted in children aged between six and 59 months in the meso-endemic area of Kenya, where the malaria prevalence rate has increased from $4 \%$ in 2007 to $8 \%$ in 2010 [28]. This high-risk population has less likelihood of semi-immunity, and hence the efficacy detected was not greatly influenced by previous exposure to malaria [29].

With widespread adoption and scaled-up use of AL dispersible and other ACT in many sub-Saharan African countries, there is a risk of development of resistance; however, so far parasite resistance has not been reported in this region. In the present study, the majority of the children had parasite clearance within 48 hours with AL and DP and the parasite clearance rate reported here was similar to previously reported rates [30,31]. This demonstrates that the $P$. falciparum parasites in this region are still sensitive to artemisinin derivatives, unlike in some regions of Southeast Asia where delayed response to ACT has been reported [32,33]. This study builds on evidence for clinical practice in Kenya and sub-Saharan African countries that AL dispersible remains efficacious as a first-line treatment for uncomplicated $P$. falciparum malaria.

Overall, both drugs were well tolerated by children. There was a comparable occurrence of AEs in the AL dispersible and DP pediatric arms, with the most commonly reported AEs being malaria and cough, in line with the previously published data [23,34-38]. No deaths were reported. One SAE was reported in the AL dispersible arm (severe malaria) and this was not considered to be related to the study drug. No discontinuations due to the study drug were observed. One limitation of the study was that it was not blinded and there was therefore potential for bias regarding tolerability and efficacy assessments. This was mitigated with a blinded randomization procedure and with the parents being blinded regarding the assigned drug.

Both of the ACT studied here are fixed-dose formulations. AL dispersible is administered twice daily for three days, whereas DP paediatric is administered once daily for three days. AL dispersible should be administered with food or milk [16] to enhance lumefantrine absorption. Although the above facts might suggest that better treatment

Table 4 Biological parameter changes during treatment

\begin{tabular}{|c|c|c|c|c|c|}
\hline \multirow[t]{2}{*}{ Parameter } & \multicolumn{2}{|c|}{ AL dispersible } & \multicolumn{2}{|c|}{ DP paediatric } & \multirow[t]{2}{*}{ p-value } \\
\hline & $\mathbf{N}$ & Mean change $(95 \% \mathrm{Cl})$ & $\mathbf{N}$ & Mean change $(95 \% \mathrm{Cl})$ & \\
\hline Haemoglobin (g/dl) & 178 & $1.6(1.3$ to 1.9$)$ & 185 & 1.7 (1.4 to 1.9$)$ & 0.134 \\
\hline White blood cells $\left(X 10^{3} / \mu \mathrm{L}\right)$ & 178 & $-0.8(-1.5$ to -0.1$)$ & 185 & $-0.5(-1.0$ to 0.3$)$ & 0.869 \\
\hline Platelets $\left(X 10^{3} / \mu \mathrm{L}\right)$ & 178 & 88.5 (74.1 to 102.8$)$ & 185 & $88.3(76.2$ to 102.8$)$ & 0.926 \\
\hline Red blood cells $\left(\times 10^{6} / \mu \mathrm{L}\right)$ & 178 & $0.6(0.4$ to 0.8$)$ & 185 & 0.7 (0.6 to 0.9$)$ & 0.394 \\
\hline Neutrophils (\%) & 178 & $-14.4(-17.2$ to -11.6$)$ & 185 & $-16.6(-19.4$ to -13.9$)$ & 0.857 \\
\hline Lymphocytes (\%) & 178 & 14.4 (11.3 to 17.4$)$ & 185 & 18.8 (16.0 to 21.6) & 0.081 \\
\hline Eosinophils (\%) & 178 & $-0.2(-0.7$ to 0.2$)$ & 185 & - & - \\
\hline Basophils (\%) & 177 & $-0.02(-0.10$ to 0.07$)$ & 185 & $-0.08(-0.19$ to 0.03$)$ & 0.309 \\
\hline Monocytes (\%) & 177 & $-0.4(-0.89$ to 0.06$)$ & 185 & $-0.78(-1.54$ to -0.02$)$ & 0.633 \\
\hline
\end{tabular}

$\mathrm{Cl}=$ confidence interval.

A negative value indicates a decrease in day 28 values, on average compared to baseline.

*p-value from ANCOVA test of difference at day 28 between arms adjusting for baseline values. 
Table 5 Drug questionnaire response

\begin{tabular}{|c|c|c|c|}
\hline & AL dispersible $N=126$ & DP paediatric $\mathrm{N}=124$ & p-value* \\
\hline Regimen, median number of tablets required & 6 & 4 & \\
\hline Pack available for survey, n (\%) & $117(93.6)$ & $107(85.6)$ & 0.089 \\
\hline \multicolumn{4}{|l|}{ Acceptability and comprehension } \\
\hline Health worker explained how to administer drug, n (\%) & $124(99.2)$ & $122(98.4)$ & 0.684 \\
\hline Health worker used pictures to explain how to administer drug, n (\%) & $123(99.2)$ & $123(99.2)$ & 0.321 \\
\hline Pictures helped in understanding how to administer, $\mathrm{n}(\%)$ & $124(99.2)$ & $124(99.2)$ & 0.159 \\
\hline \multicolumn{4}{|l|}{ Difficulty/Ease of using drug } \\
\hline Very difficult, n (\%) & 0 & $3(2.4)$ & \multirow{5}{*}{0.001} \\
\hline Difficult, n (\%) & $2(1.6)$ & $10(8.1)$ & \\
\hline Acceptable, n (\%) & $19(15.2)$ & $28(22.6)$ & \\
\hline Simple, n (\%) & $85(68.0)$ & $56(45.2)$ & \\
\hline Very simple, n (\%) & $18(14.4)$ & $27(21.8)$ & \\
\hline \multicolumn{4}{|l|}{ Difficulty/Ease of using drug A compared to B } \\
\hline Very difficult, n (\%) & - & - & \multirow{5}{*}{0.007} \\
\hline Difficult, n (\%) & $3(2.4)$ & $11(8.9)$ & \\
\hline Acceptable, n (\%) & $25(20.0)$ & $28(22.6)$ & \\
\hline Simple, n (\%) & $82(65.6)$ & $59(47.6)$ & \\
\hline Very simple, n (\%) & $14(11.2)$ & $26(21.0)$ & \\
\hline \multicolumn{4}{|l|}{ Taste of medicine for child } \\
\hline Not at all, n (\%) & 0 & $4(3.2)$ & \multirow{5}{*}{0.001} \\
\hline Not very much, n (\%) & $11(8.8)$ & $24(19.4)$ & \\
\hline Acceptable, n (\%) & $23(18.4)$ & $25(20.2)$ & \\
\hline Liked it, n (\%) & $80(64.0)$ & $50(40.3)$ & \\
\hline Liked it very much, n (\%) & $10(8.0)$ & $20(16.1)$ & \\
\hline \multicolumn{4}{|l|}{ Liquid for dissolving tablet } \\
\hline Water, n (\%) & $119(95.4)$ & $115(92.7)$ & 0.301 \\
\hline Other, n (\%) & $6(4.6)$ & $10(7.3)$ & \\
\hline Drug taken with a meal, n (\%) & $118(94.4)$ & $110(89.4)$ & \\
\hline \multicolumn{4}{|l|}{ Prefer drug given as } \\
\hline Syrup, n (\%) & $21(16.8)$ & $36(29.5)$ & \\
\hline Whole tablet which dissolves in small volume of water/milk, n (\%) & $96(76.8)$ & $76(62.3)$ & 0.025 \\
\hline Injection, n (\%) & $2(1.6)$ & $7(5.7)$ & \\
\hline Other, n (\%) & $6(4.8)$ & $8(6.4)$ & \\
\hline Prefer other anti-malarias, n (\%) & $4(3.2)$ & $5(4.0)$ & \\
\hline \multicolumn{4}{|l|}{ Perceived adverse drug reactions } \\
\hline Anything unusual after medication given, $\mathrm{n}(\%)$ & $4(3.2)$ & $1(0.8)$ & \\
\hline Concomitant medication given, $\mathrm{n}(\%)$ & $3(2.6)$ & $2(1.9)$ & \\
\hline Adverse reaction solicited, $\mathrm{n}(\%)$ & $2(1.7)$ & $1(0.9)$ & \\
\hline Caretaker reported ADR to health worker, n (\%) & $2(1.7)$ & $1(0.9)$ & \\
\hline ADR/SAE completed based on events seriousness, $n(\%)$ & $1(0.9)$ & $1(0.9)$ & \\
\hline
\end{tabular}

$\mathrm{AL}$ : artemether-lumefantrine; DP: dihydroartemisinin-piperaquine.

acceptability and tolerability may be observed with DP paediatric compared with AL dispersible, according to the survey conducted in this study, acceptability and tolerability to treatment regimen was in fact higher in the AL dispersible arm. More respondents considered AL dispersible simple to use and to have a better taste. Most ACT need to be 
crushed and mixed with water or food. Since they have a bitter taste, children can expectorate the medicine and this may result in them not receiving the full therapeutic dose. With its palatable flavour ('liked' or 'liked very much' by $72 \%$ of respondents), AL dispersible may enhance adherence and improve therapeutic outcomes in children $[39,40]$. Moreover, it has been demonstrated that with the content of standard African diets or milk optimal efficacy is achieved with fixed-dose AL (Coartem) [16]. Oral bioavalability of AL was shown to increase by $108 \%$ when a normal meal was taken close to AL dosing compared to a fasting condition. Another finding of note in the acceptability and tolerability survey was the strong preference expressed for whole tablets, which dissolve in a small volume of water/milk over syrup formulations. Previous research has shown that dry powder formulations intended for suspension in water are more often substandard, relative to tablets [41], and may contain ineffective or incorrect amounts of preservatives [42]. DP may be a good alternative to AL or may have use as rescue medication, and advice should be given regarding the policy and clinical practice for deployment of DP as second-line therapy in the treatment of uncomplicated falciparum malaria in Kenya.

\section{Conclusions}

$\mathrm{AL}$ is efficacious and remains the first-line treatment option for uncomplicated falciparum malaria. Both drugs were well tolerated by children. DP has proved to be as efficacious as AL. Parasites in this area remain sensitive to artemisinins.

\section{Competing interests}

None of the authors has affiliation to the manufacturers, and they have declared that they have no competing interests. The drugs were donated by the respective manufacturers.

\section{Authors' contributions \\ $\mathrm{BRO}$ designed the research (development of overall research plan and study oversight), conducted the research (data collection and patient care), and analysed the data. $\mathrm{KO}$ collected data, was involved in patient care and data analysis. NK collected data and was involved in patient care. JO, GO and CO designed the research plan, collected, and analysed the data. LO, DO and WA designed the research. FE and JJ carried out the molecular studies. RO and EJ designed the study and analysed the data. All authors participated in the preparation of the manuscript. All authors read and approved the final manuscript.}

\section{Acknowledgements}

This work has been published with the permission of the Director of the Kenya Medical Research Institute. This study was funded by Division of Malaria Control of the Ministry of Health, Kenya through support from WHO country office and DFID. The authors thank the patients and their parents who took part and the staff at the Ombeyi Dispensary. The authors would like to thank Ubhayabharathi Gurunath, professional medical writer (Novartis) for editorial support.

\section{Author details}

${ }^{1}$ Centre for Clinical Research, Kenya Medical Research Institute, Kisumu, Kenya. ${ }^{2}$ Walter Reed Project/Centre for Clinical Research, Kenya Medical Research Institute, Kisumu, Kenya. ${ }^{3}$ Centre for Global Health Research, Kenya Medical Research Institute, Kisumu, Kenya. ${ }^{4}$ Drugs for Neglected Disease Initiative, Kenya Medical Research Institute, Nairobi, Kenya. ${ }^{5}$ Center for Global
Health, University of New Mexico, New Mexico, USA. ${ }^{6}$ Department of Disease Control and Prevention, Ministry of Public Health and Sanitation, Nairobi, Kenya. ${ }^{7}$ Division of Malaria Control, Ministry of Public Health and Sanitation, Nairobi, Kenya.

Received: 13 August 2013 Accepted: 7 January 2014 Published: 28 January 2014

\section{References}

1. World Malaria Report: World Malaria Report; 2011. http://www.who.int/ entity/malaria/world_malaria_report_2011/9789241564403_eng.pdf.

2. World Health Organization: Guidelines for the Treatment of Malaria. 2nd edition; 2010. http://www.who.int/malaria/publications/atoz/ 9789241547925/en/index.html.

3. Dondorp AM, Nosten F, Yi P, Das D, Phyo AP, Tarning J, Lwin KM, Ariey F, Hanpithakpong W, Lee SJ, Ringwald P, Silamut K, Imwong M, Chotivanich K, Lim P, Herdman T, An SS, Yeung S, Singhasivanon P, Day NP, Lindegardh N, Socheat $D$, White NJ: Artemisinin resistance in Plasmodium falciparum malaria. N Engl J Med 2009, 361:455-67.

4. Artemisinin Resistance in Cambodia 1 (ARC1) Study Consortium, Noedl H, Se Y, Schaecher K, Smith BL, Socheat D, Fukuda MM: Evidence of artemisinin-resistant malaria in western Cambodia. N Engl J Med 2008, 359:2619-20.

5. Noedl H, Socheat D, Satimai W: Artemisinin-resistant malaria in Asia. N Engl J Med 2009, 361:540-541.

6. Rønn AM, Msangeni HA, Mhina J, Wernsdorfer WH, Bygbjerg IC: High level of resistance of Plasmodium falciparum to sulfadoxine-pyrimethamine in children in Tanzania. Trans R Soc Trop Med Hyg 1996, 90:179-81.

7. International Artemisinin Study Group, Adjuik M, Babiker A, Garner P, Olliaro P, Taylor W, White N: Artesunate combinations for treatment of malaria: meta-analysis. Lancet 2004, 363:9-17.

8. Kokwaro G, Mwai L, Nzila A: Artemether/lumefantrine in the treatment of uncomplicated falciparum malaria. Expert Opin Pharmacother 2007, 8:75-94.

9. Targett G, Drakeley C, Jawara M, von Seidlein L, Coleman R, Deen J, Pinder M, Doherty T, Sutherland C, Walraven G, Milligan P: Artesunate reduces but does not prevent posttreatment transmission of Plasmodium falciparum to Anopheles gambiae. J Infect Dis 2001, 183:1254-59.

10. Price RN, Nosten F, Luxemburger C, ter Kuile FO, Paiphun L, Chongsuphajaisiddhi T, White NJ: Effects of artemisinin derivatives on malaria transmissibility. Lancet 1996, 347:1654-58.

11. Makanga M, Bassat Q, Falade CO, Premji ZG, Krudsood S, Hunt P, Walter V, Beck HP, Marrast AC, Cousin C, Rosenthal PJ: Efficacy and safety of artemether lumefantrine in the treatment of acute uncomplicated Plasmodium falciparum malaria: a pooled analysis. Am J Trop Med Hyg 2011, 85:793-804.

12. Hamed K, Grueninger H: Coartem ${ }^{\circledR}$ : A decade of patient-centric malaria management. Expert Rev Anti Ther 2012, 10:645-659.

13. Hamel MJ, Adazu K, Obor D, Sewe M, Vulule J, Williamson JM, Slutsker L, Feikin DR, Laserson KF: A reversal in reductions of child mortality in western Kenya, 2003-2009. Am J Trop Med Hyg 2011, 85:597-605.

14. Manyando C, Mkandawire R, Puma L, Sinkala M, Mpabalwani E, Njunju E, Gomes M, Ribeiro I, Walter V, Virtanen M, Schlienger R, Cousin M, Chipimo M, Sullivan FM: Safety of artemether-lumefantrine in pregnant women with malaria: results of a prospective cohort study in Zambia. Malar J 2010, 9:249.

15. Abdulla S, Sagara I, Borrmann S, D'Alessandro U, González R, Hamel M, Ogutu B, Mårtensson A, Lyimo J, Maiga H, Sasi P, Nahum A, Bassat Q, Juma E, Otieno L, Björkman A, Beck HP, Andriano K, Cousin M, Lefèvre G, Ubben $D$, Premji Z: Efficacy and safety of artemether-lumefantrine dispersible tablets compared with crushed commercial tablets in African infants and children with uncomplicated malaria: a randomised, single-blind, multicentre trial. Lancet 2008, 372:1819-1827.

16. Premji ZG, Abdulla S, Ogutu B, Ndong A, Falade CO, Sagara I, Mulure N, Nwaiwu O, Kokwaro G: The content of African diets is adequate to achieve optimal efficacy with fixed-dose artemether-lumefantrine: a review of the evidence. Malar J 2008, 7:244.

17. Bukirwa H, Yeka A, Kamya MR, Talisuna A, Banek K, Bakyaita N, Rwakimari JB, Rosenthal PJ, Wabwire-Mangen F, Dorsey G, Staedke SG: Artemisinin combination therapies for treatment of uncomplicated malaria in Uganda. PLoS Clin Trials 2006, 1:e7. 
18. Janssens B, van Herp M, Goubert L, Chan S, Uong S, Nong S, Socheat D, Brockman A, Ashley EA, Van Damme W: A randomized open study to assess the efficacy and tolerability of dihydroartemisinin-piperaquine for the treatment of uncomplicated falciparum malaria in Cambodia. Trop Med Int Health 2007, 12:251-259.

19. Nambozi M, Van Geertruyden JP, Hachizovu S, Chaponda M, Mukwamataba $D$, Mulenga M, Ubben D, D'Alessandro U: Safety and efficacy of dihydroartemisinin-piperaquine versus Artemether-lumefantrine in the treatment of uncomplicated Plasmodium falciparum malaria in Zambian children. Malar J 2011, 10:50.

20. ICH Harmonized Tripartite guideline: Guidelines for Good Clinical practiceE6 (R1);1996. Current Step 4 version. http://www.ich.org/fileadmin/ Public_Web_Site/ICH_Products/Guidelines/Efficacy/E6_R1/Step4/ E6_R1__Guideline.pdf.

21. World Health Organization, Communicable Diseases Cluster: Severe falciparum malaria. Trans R Soc Trop Med Hyg 2000, 94(1):S1-90.

22. World Health Organization: Assessment and monitoring of antimalarial drug efficacy for the treatment of uncomplicated falciparum malaria. Geneva: WHO. WHO/HTM/RBM/2003.50 2003.

23. Kamya MR, Yeka A, Bukirwa H, Lugemwa M, Rwakimari JB, Staedke SG, Talisuna AO, Greenhouse B, Nosten F, Rosenthal PJ, Wabwire-Mangen F, Dorsey G: Artemether-lumefantrine versus dihydroartemisininpiperaquine for treatment of malaria: a randomized trial. PLoS Clin Trials 2007, 2:e20.

24. Wabwire-Mangen F, Bukirwa $H$, Rwakimari JB, Staedke SG, Rosenthal PJ, Lugemwa M, Yeka A, Dorsey G, Kamya MR, Talisuna A: Artemetherlumefantrine versus dihydroartemisinin-piperaquine for treating uncomplicated malaria: a randomized trial to guide policy in Uganda. PLoS One 2008, 3:e2390.

25. Ezzet F, van Vugt M, Nosten F, Looareesuwan S, White $\mathrm{NJ}$ : Pharmacokinetics and pharmacodynamics of lumefantrine (benflumetol) in acute falciparum malaria. Antimicrob Agents Chemother 2000, 44:697-704

26. Product monograph; 2010. [http://www.coartem.com/product-monograph. htm] Coartem ${ }^{\oplus}$-artemether/lumefantrine.

27. Hung TY, Davis TM, llett KF, Karunajeewa H, Hewitt S, Denis MB, Lim C, Socheat D: Population pharmacokinetics of piperaquine in adults and children with uncomplicated falciparum or vivax malaria. $\mathrm{Br} J \mathrm{Clin}$ Pharmacol 2004, 57:253-262

28. Badu K, Afrane YA, Larbi J, Stewart VA, Waitumbi J, Angov E, Ong'echa JM, Perkins DJ, Zhou G, Githeko A, Yan G: Marked variation in MSP-119 antibody responses to malaria in western Kenyan highlands. BMC Infect Dis 2012, 12:50.

29. Kenya malaria indicator survey (DoMC); 2010. http://measuredhs.com/pubs/ pdf/MIS7/MIS7.pdf.

30. Elamin SB, Awad Al, Eltayeb IB, Elmardi KA, Hassan AH, Mohamed AO, Malik EM, Mohamad TA: Descriptive study on the efficacy of artemetherlumefantrine in the treatment of uncomplicated Plasmodium falciparum malaria in Sudan. Eur J Clin Pharmacol 2010, 66:231-237.

31. Juma EA, Obonyo CO, Akhwale WS, Ogutu BR: A randomized, open-label, comparative efficacy trial of Artemether-lumefantrine suspension versus Artemether-lumefantrine tablets for treatment of uncomplicated Plasmodium falciparum malaria in children in western Kenya. Malar J 2008, 7:262.

32. Resistance to artemisinin derivatives along the Thai-Cambodian border. Wkly Epidemiol Rec 2007, 82:360.

33. WHO: The status of drug-resistant malaria along the Thailand-Myanmar border. http://www.who.int/malaria/publications/atoz/ drug_resistance_myanmar_thailand_border_may_2012.pdf.

34. Arinaitwe E, Sandison TG, Wanzira H, Kakuru A, Homsy J, Kalamya J, Kamya MR, Vora N, Greenhouse B, Rosenthal PJ, Tappero J, Dorsey G: Artemetherlumefantrine versus dihydroartemisinin-piperaquine for falciparum malaria: a longitudinal, randomized trial in young Ugandan children. Clin Infect Dis 2009, 49:1629-1637.

35. Ratcliff A, Siswantoro H, Kenangalem E, Maristela R, Wuwung RM, Laihad F, Ebsworth EP, Anstey NM, Tjitra E, Price RN: Two fixed-dose artemisinin combinations for drug-resistant falciparum and vivax malaria in Papua, Indonesia: an open-label randomised comparison. Lancet 2007, 369:757-765.

36. Bassat $Q$, Mulenga $M$, Tinto $H$, Piola $P$, Borrmann $S$, Menéndez $C$, Nambozi M, Valéa I, Nabasumba C, Sasi P, Bacchieri A, Corsi M, Ubben D, Talisuna A,
D'Alessandro U: Dihydroartemisinin-piperaquine and artemetherlumefantrine for treating uncomplicated malaria in African children: a randomised, non-inferiority trial. PLOS One 2009, 4:e7871.

37. Zwang J, Ashley EA, Karema C, D'Alessandro U, Smithuis F, Dorsey G, Janssens B, Mayxay M, Newton P, Singhasivanon P, Stepniewska K, White NJ, Nosten F: Safety and efficacy of dihydroartemisinin-piperaquine in falciparum malaria: a prospective multi-centre individual patient data analysis. PLoS One 2009, 4:e6358.

38. Myint HY, Ashley EA, Day NP, Nosten F, White NJ: Efficacy and safety of dihydroartemisinin-piperaquine. Trans R Soc Trop Med Hyg 2007, 101:858-866.

39. Abdulla S, Sagara I: Dispersible formulation of artemether/lumefantrine: specifically developed for infants and young children. Malar J 2009, 8(1):S7.

40. Makanga M, Krudsood S: The clinical efficacy of artemether/lumefantrine (Coartem). Malar J 2009, 8(1):S5.

41. Atemnkeng MA, De Cock K, Plaizier-Vercammen J: Quality control of active ingredients in artemisinin-derivative antimalarials within Kenya and DR Congo. Trop Med Int Health 2007, 12:68-74.

42. Atemnkeng MA, De Cock K, Plaizier-Vercammen J: Post-marketing assessment of content and efficacy of preservatives in artemisinin-derived antimalarial dry suspensions for paediatric use. Malar J 2007, 6:12

\section{doi:10.1186/1475-2875-13-33}

Cite this article as: Ogutu et al:: Efficacy and safety of artemetherlumefantrine and dihydroartemisinin-piperaquine in the treatment of uncomplicated Plasmodium falciparum malaria in Kenyan children aged less than five years: results of an open-label, randomized, single-centre study. Malaria Journal 2014 13:33.

\section{Submit your next manuscript to BioMed Central and take full advantage of:}

- Convenient online submission

- Thorough peer review

- No space constraints or color figure charges

- Immediate publication on acceptance

- Inclusion in PubMed, CAS, Scopus and Google Scholar

- Research which is freely available for redistribution

Submit your manuscript at www.biomedcentral.com/submit
C Biomed Central 